\title{
MODELLING OF THE VIBRATION EXPOSURE IN TYPICAL WORKING MACHINES BY MEANS OF RANDOM INPUT SIGNALS
}

\author{
IgOR MaCIEJEWski, TOMASZ KRZYŻYŃSKI \\ Koszalin University of Technology, Faculty of Technology and Education, Koszalin, Poland \\ e-mail: igor.maciejewski@tu.koszalin.pl; tomasz.krzyzynski@tu.koszalin.pl
}

\begin{abstract}
The aim of the paper is to formulate a generalized methodology of modelling random vibrations that are experienced by machine operators during their work. In the following paper, spectral characteristics of input vibrations are specified in such a way that the generated excitation signals are representative for different types of working machines. These signals could be used for determination and evaluation of risks from exposure to whole-body vibration.
\end{abstract}

Keywords: simulated input vibration, random vibration

\section{Introduction}

There are two basic sources of mechanical vibrations that can disturb proper functioning of machines. The first one relates to systems that during their operation generate vibrations, e.g. engine vibrations in the working machine (Preumont, 2002). The second class concerns systems that vibrate due to external factors, e.g. cab vibrations of the machine that is moving over uneven ground (Maciejewski and Krzyżyński, 2011; Nabaglo et al., 2013). Many sources of vibrations can cause periodic or random reactions of machine elements (Sapiński and Rosół, 2007; Maślanka et al., 2007). In such a situation, resonant states can be obtained and they may cause disturbances in motion of individual machine elements. Resonant vibrations have an adverse effect on machine functioning, and this can lead to their failure. In addition, vibrations have a negative influence on health of operators of working machines.

Machine operators during their work are exposed to vibrations that are caused very often by motion of machinery over uneven ground (Kowal et al., 2008; Snamina et al., 2013). In the case of whole-body vibration, the seat of a vehicle or the platform of a worker vibrates and this motion is transmitted into the human body. Under normal operating conditions, humans occupy the following body positions (Griffin et al., 2006):

- sitting position (mechanical vibrations transmitted through pelvis) that precludes active damping of vibration using lower limbs,

- standing position (mechanical vibrations transmitted through feet) that allows active damping of vibration in the low frequency range.

The basic positions of the human body at work are presented in Fig. 1. Harmful vibrations can be transmitted to the human body in three orthogonal directions: longitudinal $x$, lateral $y$ and vertical $z$. Vibrations are defined by their magnitudes and frequencies. The magnitudes of vibration are traditionally expressed as vibration acceleration, because most vibration transducers produce an output that is related to the acceleration signal. Frequencies appearing in the random whole-body vibration environment usually occur between 1 and $20 \mathrm{~Hz}$ (Krzyzynski et al., 2004). Exposure to whole-body vibration causes motions and forces within the human body that may cause discomfort, adversely affect working performance and cause health and safety risk (Griffin et al., 2006). 


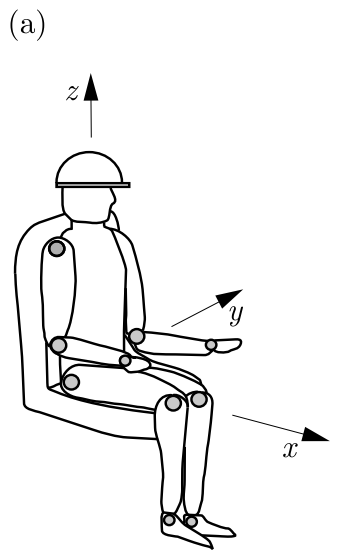

(b)

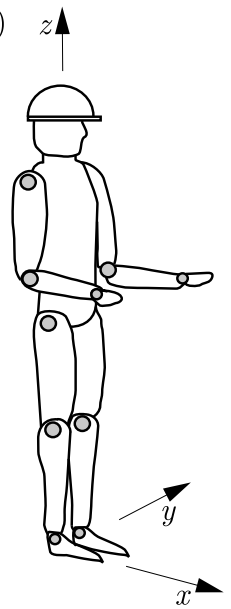

Fig. 1. Positions of the human body at work: sitting position (a), standing position (b)

The basic opportunity to minimise harmful vibrations consists in applying a vibration reduction system that prevents propagation of mechanical vibrations from their source to the isolated body (Kowal et al., 2008). Dynamic characteristics of a vibration isolator should be selected for a specific application in such a way that the vibrations transmitted from the source to the body are minimal (Snamina et al., 2013). In order to analyze dynamics of a vibration isolation system, the excitation signal with specific spectral characteristics should be used. Unfortunately, there are no effective methods for generating signals representing the working of different machinery. In the papers [3], [7], [8], the target spectral characteristics are only standardised for the simulated input vibration test in the vertical direction. There is a lack of effective procedures of generating the time history of random signals with precisely defined spectral properties. In addition, it is not known how to reproduce the excitation signals based on the measured spectral characteristics (Bluthner et al., 2008), especially in horizontal directions (lateral and longitudinal).

In the following paper, an effective method of reproducing random vibration in different types of working machines is proposed for the purpose of selecting dynamic characteristics of vibration reduction systems. At first, machines having similar operational vibration are grouped by virtue of various mechanical characteristics. Then a signal generator is utilized to generate random vibration and an original signal processing technique is used in order to obtain the spectral characteristics representing vibration in a specific group of machines.

\section{Modelling of the vibration reduction system}

The general model of the vibration reduction system used in typical working machines is presented in Fig. 2. The suspended body is isolated against harmful vibrations in three orthogonal directions: longitudinal $x$, lateral $y$ and vertical $z$. The passive visco-elastic elements are used in order to minimise the human exposure to whole-body vibration. Rotation vibrations around each axis of the Cartesian coordinate system $(x, y, z)$ are neglected in this simplified model. Ongoing research (Maciejewski et al., 2011) indicates that the exposure of workers to risks arising from vibration are evaluated for translational axes, therefore in this paper, vibro-isolation properties are discussed for this specific direction. The equation of motion for such a system is formulated in the matrix form

$$
\mathbf{M}_{i} \ddot{\mathbf{q}}_{i}+\mathbf{D}_{i} \dot{\mathbf{q}}_{i}+\mathbf{C}_{i} \mathbf{q}_{i}=\mathbf{F}_{s i} \quad i=x, y, z
$$


where $\mathbf{q}_{i}$ is the displacement vector of the isolated body, $\mathbf{M}_{i}, \mathbf{D}_{i}, \mathbf{C}_{i}$ are the inertia, damping and stiffness matrices, respectively, $\mathbf{F}_{s i}$ is the vector of exciting forces describing the non-linear vibration isolator.

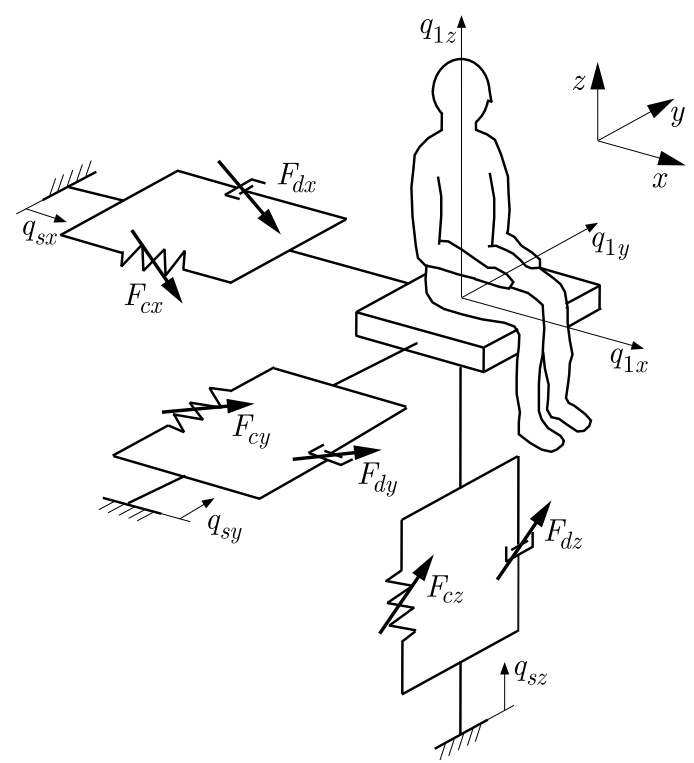

Fig. 2. General model of the non-linear vibration reduction system used in typical working machines

There are dozens of human body models presented in the modern literature (Rutzel et al., 2006; Stein et al., 2007; Toward and Griffin, 2011). Usually, there are multi-degree of freedom lumped parameter models that consider the seating and standing position. In this paper, the generalised mathematical model of vibration reduction system is presented that allows one to use various bio-mechanical structures of the well-known human body models. The $n$-element vector $\mathbf{q}_{i}$ represents the movement of elements contained in the bio-mechanical model of the human body

$$
\mathbf{q}_{i}=\left[q_{1 i}, q_{2 i}, \ldots, q_{n i}\right]^{\mathrm{T}} \quad i=x, y, z
$$

where: $q_{1 i}, q_{2 i}, \ldots, q_{n i}$ are displacements of the human body model. The $n$-element vector of exciting forces $\mathbf{F}_{s i}$ is given by the following expression

$$
\mathbf{F}_{s i}=\left[F_{s i}, 0, \ldots, 0\right]^{\mathrm{T}} \quad i=x, y, z
$$

The particular non-linear exciting forces $F_{s i}$ can be described in a general form as follows

$$
F_{s i}=\sum_{k=1}^{l} F_{d i k}\left(\dot{q}_{1 i}-\dot{q}_{s i}\right)+\sum_{k=1}^{l} F_{c i k}\left(q_{1 i}-q_{s i}\right) \quad i=x, y, z
$$

where: $F_{c i k}\left(q_{1 i}-q_{s i}\right)$ are non-linear functions including force characteristics of the conservative elements as a function of the system relative displacement $q_{1 i}-q_{s i}, F_{d i k}\left(\dot{q}_{1 i}-\dot{q}_{s i}\right)$ are non-linear functions including force characteristics of the dissipative elements as a function of the system relative velocity $\dot{q}_{1 i}-\dot{q}_{s i}$. These non-linear characteristics should be selected especially for well-defined input vibrations by using appropriate element models. Exemplary models can be found in the authors' previous paper (Maciejewski et al., 2011). The input displacement $q_{s i}$ and velocity $\dot{q}_{s i}$ are modelled as discrete-time random signals that are generated for the specific direction of vibration exposure $(i=x, y, z)$.

Modelling of the random vibration acceleration $\ddot{q}_{s i}(t)$ occurring in typical working machines is presented in the next part of the following paper. The elaborated signal models allow one to 
test a variety of vibration reduction systems by means of the laboratory measurements and/or numerical simulations. The proposed method can be used for generating signals specified by the present test standards, see [3], [7], [8] and also for reproducing signals measured by other authors (Bluthner et al., 2008). The requirements regarding limitations of the vibration simulator (shaker) can be found in International Standards ([3], [7], [8]). Safety requirements for the test person are exhaustively discussed in ISO-2613 [6].

\section{Random signal generator}

The simulated input vibration is generated using a normally (Gaussian) distributed random numbers (rand) that produces a waveform

$$
\ddot{q}_{s i j}(t)=\sqrt{\sigma_{s i j}^{2}} \operatorname{rand}\left(\frac{t_{k}}{t_{s}}\right) \quad i=x, y, z \quad j=1, \ldots, l
$$

where: $q_{s i j}(t)$ is vibration acceleration generated in the direction $x, y, z, j=1, \ldots, l$ is the number of signal generator, $\sigma_{s i j}^{2}$ is the variance of random numbers, $t$ is the current time instant, $t_{k}$ is the computation time, $t_{s}$ is the time interval between samples.

In order to simulate the effect of white noise with a correlation time near 0 and a flat power spectral density, a sampling time should be much smaller than the fastest dynamics of the system. For intended analysis with high resolution, an acceptable random signal can be achieved by specifying the sampling frequency 100 times (or more) of the maximal frequency of the system to be tested. Therefore, the time interval between samples of the random signal is $t_{s}=0.01 / f_{\max }$, where $f_{\max }$ is the maximal frequency of the system in Hz. For generation of vibration up to $20 \mathrm{~Hz}$, the maximal frequency of the random signal is set to $25 \mathrm{~Hz}$.

The probability density function of a normally distributed random signal can be described using the following relation

$$
p\left(\ddot{q}_{s i j}(t)\right)=\frac{1}{\sqrt{2 \pi \sigma_{s i j}^{2}}} \exp \left(-\frac{1}{2 \sigma_{s i j}^{2}} \ddot{q}_{s i j}^{2}(t)\right) \quad i=x, y, z \quad j=1, \ldots, l
$$

The time plot of a stochastic signal $\ddot{q}_{s i j}(t)$ is shown in Fig. 3a, and its histogram on the background of the probability density function is illustrated in Fig. 3b.

(a)

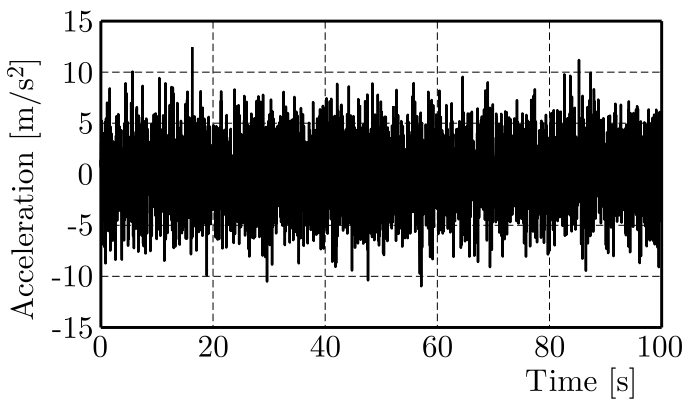

(b)

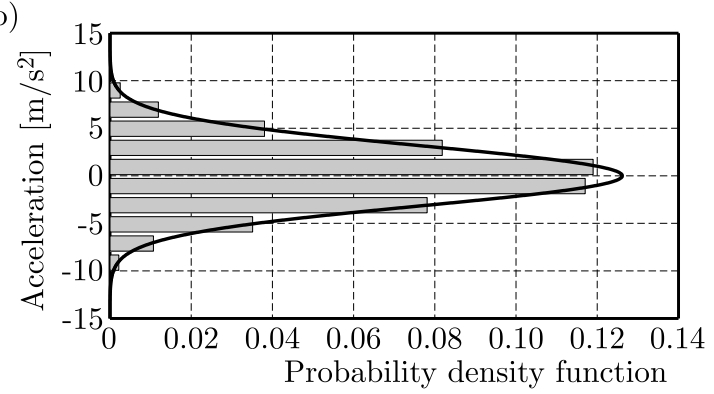

Fig. 3. Input vibration generated as a normally distributed random process (a) and its histogram (grey box) on the background of the probability density function (black line) (b)

The spectral properties of the generated random signal have to be close to white noise, so the power spectral density (PSD) should be flat in the considered frequency range, and the correlation time of a time series shall be close to zero (Bendat and Piersol, 2004). The power spectral density and the normalized auto-correlation of the generated signal are presented in Fig. 4. 

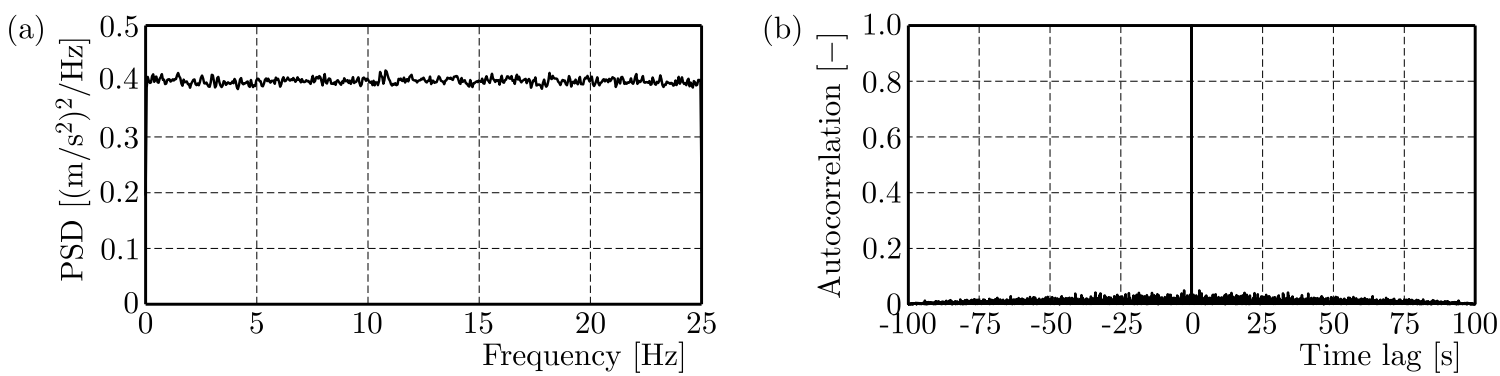

Fig. 4. Power spectral density (a) and normalized autocorrelation (b) of the generated random signal

\section{Signal processing technique}

An original signal processing technique is proposed in order to obtain specific spectral properties of the generated random signal. This technique involves the making use of a set of the Butterworth filters (high-pass and low-pass). A block diagram of the proposed signal processing technique is shown in Fig. 5.

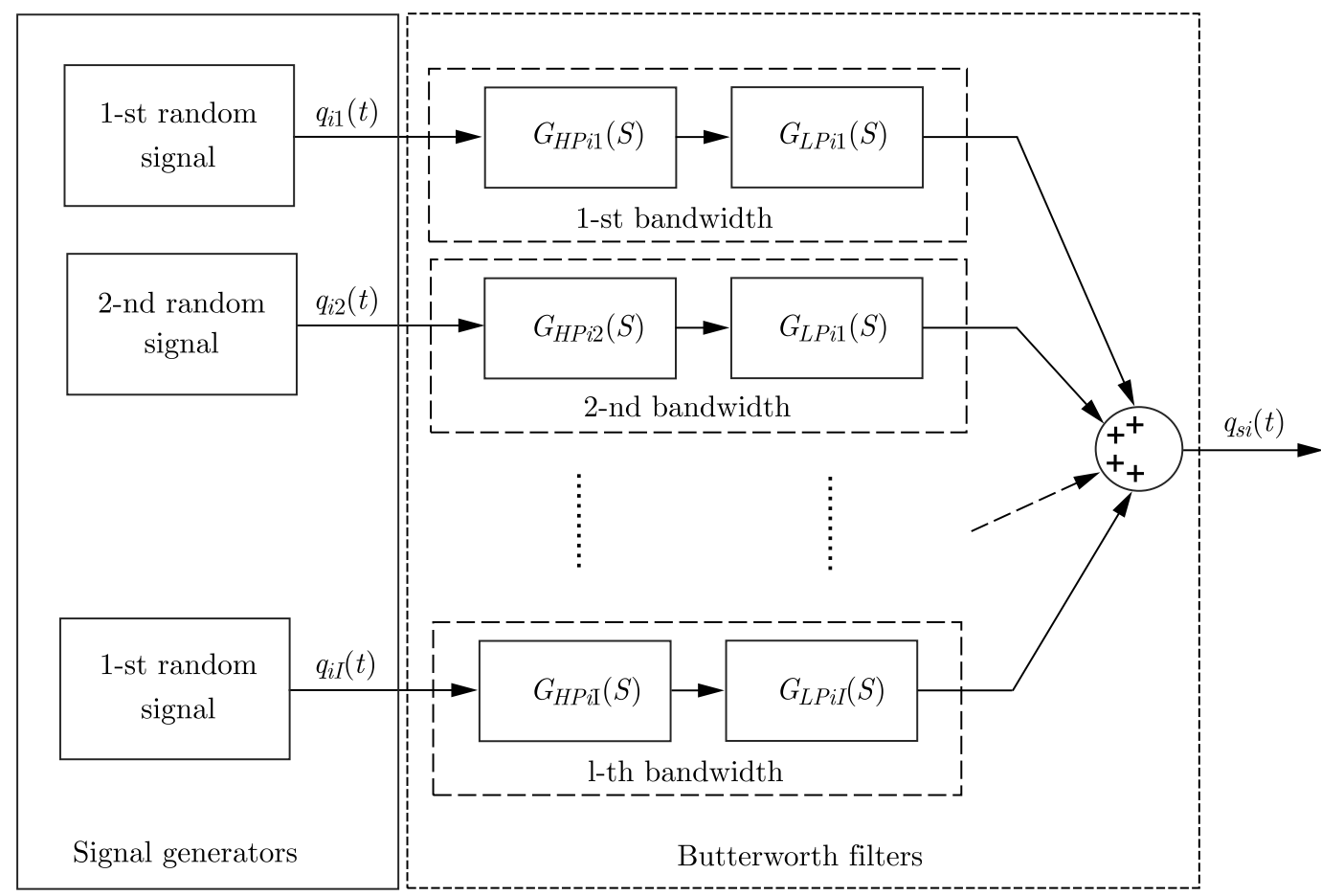

Fig. 5. Block diagram of the proposed signal processing technique

Transfer functions for the linear Butterworth filters are defined in the following way (Parks and Burrus, 1987):

- high-pass filter

$$
G_{H P s i j}(s)=\frac{s^{n}}{s^{n}+a_{n-1} s^{n-1}+\ldots+a_{1} s+1} \quad i=x, y, z \quad j=1, \ldots, l
$$

— low-pass filter

$$
G_{L P s i j}(s)=\frac{1}{s^{n}+a_{n-1} s^{n-1}+\ldots+a_{1} s+1} \quad i=x, y, z \quad j=1, \ldots, l
$$

where: $a_{1}$ to $a_{n}$ are the coefficients of the Butterworth filter specified in Table $1, n$ is the order of the filter, $s$ is the Laplace variable, $j=1, \ldots, l$ is the number of filters used. 
Table 1. Coefficients of the Butterworth filter $a_{1}, \ldots, a_{n}$ for different filter orders $n$

\begin{tabular}{|c|c|c|c|c|c|c|c|}
\hline$n$ & $a_{1}$ & $a_{2}$ & $a_{3}$ & $a_{4}$ & $a_{5}$ & $a_{6}$ & $a_{7}$ \\
\hline \hline 2 & 1.414 & - & - & - & - & - & - \\
\hline 3 & 2.000 & 2.000 & - & - & - & - & - \\
\hline 4 & 2.613 & 3.414 & 2.613 & - & - & - & - \\
\hline 5 & 3.236 & 5.236 & 5.236 & 3.236 & - & - & - \\
\hline 6 & 3.863 & 7.464 & 9.141 & 7.464 & 3.863 & - & - \\
\hline 7 & 4.493 & 10.097 & 14.591 & 14.591 & 10.097 & 4.493 & - \\
\hline 8 & 5.125 & 13.137 & 21.846 & 25.688 & 21.846 & 13.137 & 5.125 \\
\hline
\end{tabular}

The high-pass and low-pass filters (Eqs. (4.1) and (4.2)) allows one to create the required band-pass filter for a specified frequency bandwidth. A combination of the high-pass and low-pass filters at the defined cut-off frequency and filter order provides generating of the input vibration with various spectral characteristics. The transfer function of the total filter is defined as follows

$$
G_{s i}(s)=\frac{N_{s i}(s)}{D_{s i}(s)}=\sum_{j=1}^{l} G_{H P s i j}(s) G_{L P s i j}(s) \quad i=x, y, z \quad j=1, \ldots, l
$$

where: $N_{s i}(s)$ and $D_{s i}(s)$ are the numerator and denominator polynomials that represent a combination of the Butterworth filters shown in Fig. 5.

The transfer function (Eq. (4.3)) is especially useful when analyzing the proposed filter stability. If all poles of this transfer function have negative real parts, then the filter is considered as asymptotically stable. However, the filter is unstable when any pole has a positive real part. Therefore, all poles in the complex $s$-plane should be located in the left half plane to ensure filter stability, which can be described by the following relation

$$
\operatorname{Re}\left(D_{s i}(s)\right)<0 \quad i=x, y, z
$$

Pole distribution in the complex $s$-plane for unstable and stable filter is shown in Fig. 6.
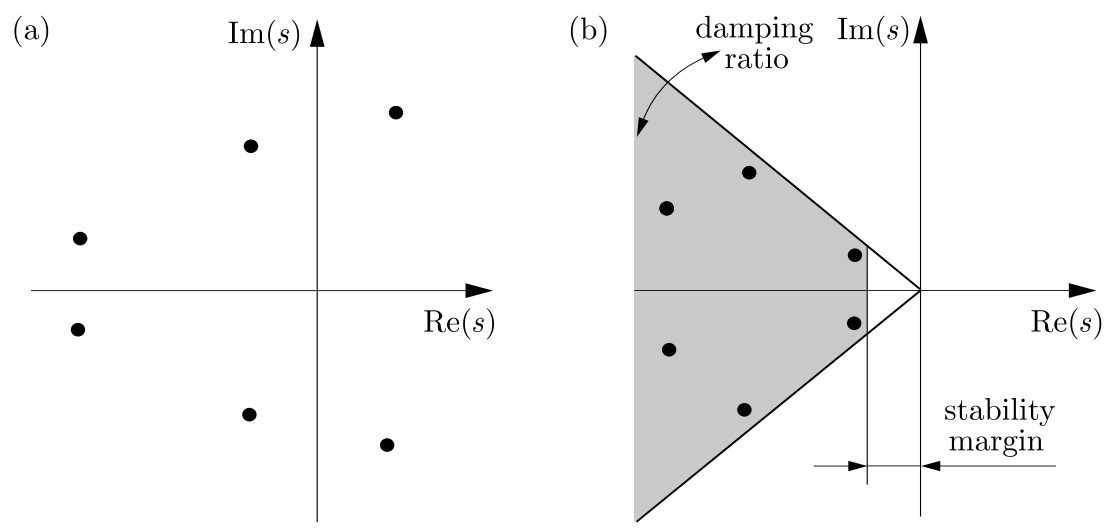

Fig. 6. Pole distribution in the complex s-plane: unstable filter (a), stable filter with stability margin (b)

The input vibration is defined by a power spectral density of the vertical acceleration and by the root mean square value of the acceleration signal. The target magnitude $\operatorname{PSD}_{s i}(2 \pi f)$ of the power spectral density function can be calculated with the assistance of the following equation

$$
\operatorname{PSD}_{s i}(2 \pi f)=\sum_{j=1}^{l} \frac{2 \sigma_{s i j}^{2}}{f_{s}}\left|\left(G_{H P s i j}(2 \pi f)\right)^{2}\left(G_{L P s i j}(2 \pi f)\right)^{2}\right| \quad \begin{aligned}
& i=x, y, z \\
& j=1, \ldots, l
\end{aligned}
$$


where: $f$ is the frequency in $\mathrm{Hz}, \sigma_{s i j}^{2}$ is the signal variance in a specified frequency bandwidth, $f_{s}$ is the sampling frequency. The curve defined by Eq. (4.5)) consists of target power spectral density to be produced for the simulated input vibration (Fig. 7).

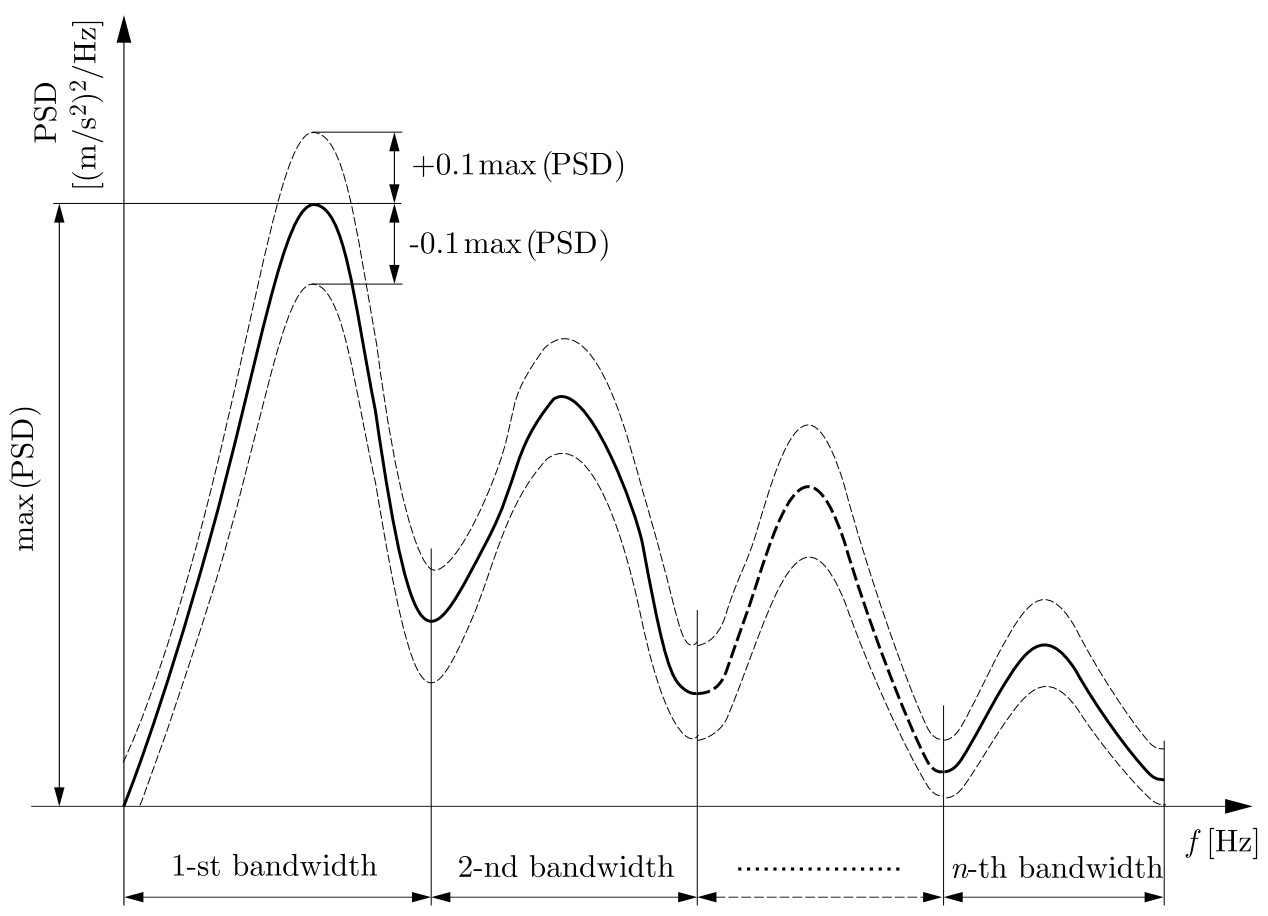

Fig. 7. Target power spectral density of the simulated input vibration

The power spectral density of the simulated acceleration signal is considered to be representative for different types of working machines if and only if ([3], [7], [8]):

- the magnitude of simulated input is within the tolerance of the target power spectral density function $\operatorname{PSD}_{s i}(2 \pi f) \pm 0.1 \max _{f}\left(\operatorname{PSD}_{s i}(2 \pi f)\right)$,

- the root mean square (RMS) value of simulated input acceleration is within the tolerance of the required value $\left(\ddot{q}_{s i}\right)_{R M S} \pm 0.05\left(\ddot{q}_{s i}\right)_{R M S}$.

The root mean square value of the acceleration signal is defined using the following relation

$$
\left(\ddot{q}_{s i}\right)_{R M S}=\sqrt{\frac{1}{t_{k}} \int_{0}^{t_{k}}\left(\ddot{q}_{s i}(t)\right)^{2} d t} \quad i=x, y, z
$$

where: $\ddot{q}_{s i}(t)$ is the time history of input vibration and $t_{k}$ is the duration within which vibration data for analysis is obtained.

\section{Spectral estimation method}

A novel method is proposed in order to evaluate parameters (signal variances and filter cut-off frequencies) of the system presented in Fig. 5. Such a parametric method allows one to find the system configuration with a magnitude response approximating the desired function (Fig. 8). Therefore, a random signal with the user-defined spectral properties can easily be generated. This is the essence of the importance and novelty of the authors' method in comparison with the spectral estimation methods used by most authors. The proposed method applies the least square error (LSE) minimization technique over the frequency range of the filter response. 


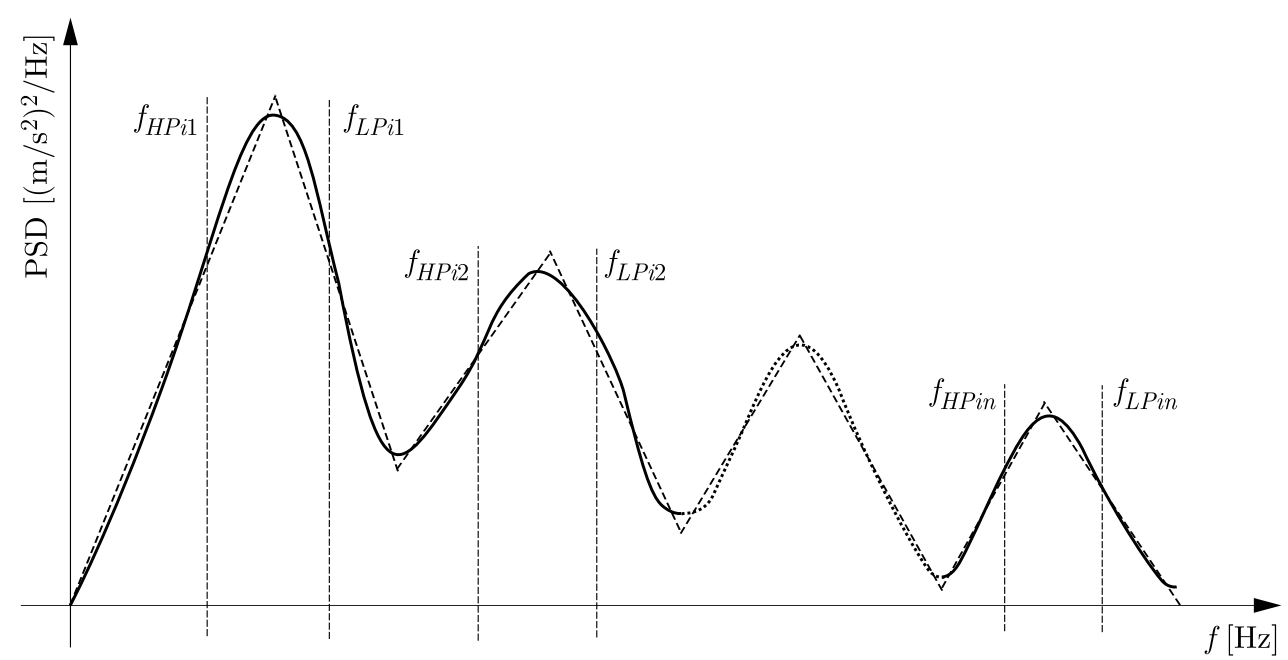

Fig. 8. Magnitude response (-) approximating the desired function (- - -)

The least square error of the specified frequency response is described in the following form

$$
\operatorname{LSE}_{s i}=\sqrt{\sum_{k=1}^{m}\left(\operatorname{PSD}_{s i}\left(2 \pi f_{k}\right)-\widehat{\operatorname{PSD}}_{s i}\left(2 \pi f_{k}\right)\right)^{2}} \quad i=x, y, z
$$

where: $\mathrm{PSD}_{s i}$ and $\widehat{\mathrm{PSD}}_{s i}$ are the desired and estimated power spectral densities that are obtained for the same frequency value $f_{k}$.

The error minimization includes design parameters having an influence on the spectral characteristics of the generated signal as follows

$$
\min _{\sigma_{s i j}^{2}, f_{H P s i j}, f_{L P s i j}} \operatorname{LSE}_{s i}\left(\sigma_{s i j}^{2}, f_{H P s i j}, f_{L P s i j}\right) \quad i=x, y, z \quad j=1, \ldots, l
$$

where: $\sigma_{s i j}^{2}$ is the variance of the random signal, $f_{H P s i j}$ and $f_{L P s i j}$ are the cut-off frequencies of the high-pass and low-pass Butterworth filters, respectively.

The problem of minimizing the objective function (Eq. (5.2)) of several parameters (decision variables) is defined subject to linear inequality constraints on these variables

$$
\begin{aligned}
& \left(\sigma_{\text {sij }}^{2}\right)_{\min } \leqslant \sigma_{\text {sij }}^{2} \leqslant\left(\sigma_{\text {sij }}^{2}\right)_{\max } \\
& \left(f_{H P s i j}\right)_{\min } \leqslant f_{H P s i j} \leqslant\left(f_{H P s i j}\right)_{\max } \\
& \left(f_{L P s i j}\right)_{\min } \leqslant f_{L P s i j} \leqslant\left(f_{L P s i j}\right)_{\max }
\end{aligned} \quad i=x, y, z \quad j=1, \ldots, l
$$

where: $\left(\sigma_{s i j}^{2}\right)_{\min }$ and $\left(\sigma_{s i j}^{2}\right)_{\max }$ are the lowest and highest value of the signal variance, $\left(f_{H P s i j}\right)_{\min }$ and $\left(f_{H P s i j}\right)_{\max }$ are the lowest and highest value of the cut-off frequencies of the high-pass filter, $\left(f_{L P s i j}\right)_{\min }$ and $\left(f_{L P s i j}\right)_{\max }$ are the lowest and highest value of the cut-off frequencies of the low-pass filter.

Additionally, nonlinear inequality constraints are imposed on the objective function (Eq. (5.2)) to restrict the filter cut-off frequencies in the following order

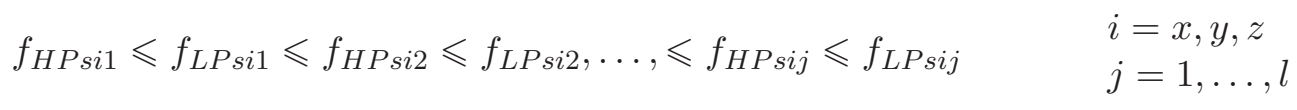

where: $j=1, \ldots, l$ is the number of low-pass (LP) and high-pass (HP) filters used to estimate the input vibration in different directions $(i=x, y, z)$. 
The multiple correlation coefficient $(R)$ is employed in order to measure how successful is the approximation in explaining the variation of the optimisation results. This coefficient is defined as follows

$$
R_{s i}=\sqrt{1-\frac{\sum_{k=1}^{m}\left(\widehat{\mathrm{PSD}}_{s i}\left(2 \pi f_{k}\right)-\mathrm{PSD}_{s i}\left(2 \pi f_{k}\right)\right)^{2}}{\sum_{k=1}^{m}\left(\mathrm{PSD}_{s i}\left(2 \pi f_{k}\right)-\overline{\mathrm{PSD}}_{s i}\left(2 \pi f_{k}\right)\right)^{2}}} \quad i=x, y, z
$$

where: $\widehat{\mathrm{PSD}}_{s i}$ is the estimated power spectral density for the directions $x, y, z, \mathrm{PSD}_{s i}$ is the desired power spectral density, $\overline{\mathrm{PSD}}_{s i}$ is the mean value of the desired power spectral density, $f_{k}$ is the discrete value of frequency. The coefficient $R_{s i}$ can take any value between 0 and 1 , with a value closer to 1 indicating a better estimation of the power spectral density.

\section{Vertical vibration}

The standards ([3], [7], [8]) specify the laboratory simulated vertical vibration ( $z$-axis) that is based on representative measured data from different types of machines in typical working conditions. The input spectral classes are defined for machines having similar mechanical characteristics. The test inputs indicated in these standards are based on a large number of measurements taken in situ of working machines while they were used under severe operating conditions.

International Standard ISO 7096 [7] specifies input vibrations for earth-moving machinery in nine spectral classes (EM1-EM9). British Standard BS EN 13490 [3] defines the input spectral classes required for industrial trucks (IT1-IT4). ISO 5007 standard [8] specifies input vibration in three input spectral classes (AG1-AG3) for agricultural tractors with rubber tyres, unsprung rear axles and no low-frequency cab isolation. Each class defines a group of machines having similar vibration characteristics.

All of these standards completely designate high-pass and low-pass filters of the Butterworth type that are required for generating vibration along the vertical axis. Numerical values of the cut-off frequencies and filter orders are presented in Table 2. The vibration characteristics for selected input spectral classes, i.e. EM1, EM5-EM7, IT2-IT3, AG1-AG2 and their tolerances, are shown in Fig. 9. The desired and obtained root mean square values of the acceleration signal are defined in Table 2.

Table 2. Parameters of the input vibration generated for different types of working machines

\begin{tabular}{|c|c|c|c|c|c|c|c|c|c|c|c|c|c|c|c|c|c|}
\hline & \multirow{2}{*}{\multicolumn{3}{|c|}{\begin{tabular}{|l|} 
Signal generators \\
$\sigma^{2}\left[\left(\mathrm{~m}^{2}\right)^{2}\right]$ \\
\end{tabular}}} & \multicolumn{5}{|c|}{ High-pass filters } & \multicolumn{5}{|c|}{ Low-pass filters } & \multicolumn{2}{|c|}{ Results } \\
\hline & & & & & & \multirow{2}{*}{\multicolumn{2}{|c|}{$\frac{f_{H P s i j}}{j}$}} & \multirow[t]{2}{*}{$\mathrm{Hz}]$} & \multicolumn{2}{|c|}{$n_{H P s i j}$} & \multicolumn{3}{|c|}{$f_{L P s i j}[\mathrm{~Hz}]$} & \multicolumn{2}{|c|}{$n_{L P s i j}$} & \multicolumn{2}{|c|}{$\left(\ddot{q}_{s i}\right)_{R M S},\left[\frac{\mathrm{m}}{\mathrm{s}}^{2}\right.$} \\
\hline \multirow{2}{*}{$\begin{array}{c}\text { Type of } \\
\text { machines }\end{array}$} & \multirow{2}{*}{\begin{tabular}{c|} 
Input \\
vibration
\end{tabular}} & & \multicolumn{3}{|c|}{$\frac{\sigma_{s i j}^{2},\left[\left(\frac{\mathrm{m}^{2}}{\mathrm{~s}}\right)^{2}\right]}{j}$} & & & & $\bar{j}$ & & & $j$ & & & & Desi- & Obta- \\
\hline & & & 1 & 2 & 3 & 1 & 2 & 3 & \begin{tabular}{|l|l|}
1 & 2 \\
\end{tabular} & 3 & 1 & 2 & 3 & \begin{tabular}{|l|l|}
1 & 2 \\
\end{tabular} & 3 & red & ined \\
\hline Earth & EM1 & $\mathrm{z}$ & 282 & - & - & 1.5 & 5 & $=$ & 4 & - & 2.5 & $=$ & $\overline{-}$ & \begin{tabular}{|l|l}
4 & - \\
-1
\end{tabular} & - & 1.71 & 1.67 \\
\hline moving & EM5 & $\mathrm{z}$ & 111 & - & - & 1.5 & - & - & $4 \mid$ & - & 3.5 & - & - & 1 & - & 1.94 & 2.10 \\
\hline machinery & EM6 & $\mathrm{z}$ & 79 & - & - & 6.5 & - & - & 2 & - & 9 & - & - & $2 \mid$ & - & 1.65 & 1.70 \\
\hline & EM7 & $\mathrm{z}$ & 925 & - & & 3 & - & - & 8 & & 3.5 & - & & 8 & & 2.25 & 2.27 \\
\hline Industrial & IT2 & $\mathrm{z}$ & 145 & - & - & 3 & - & - & 4 & 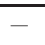 & 3 & - & - & $2-$ & 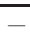 & 1.05 & 1.05 \\
\hline trucks & IT3 & $\mathrm{z}$ & 60 & - & - & 1.5 & - & - & 4 & - & 3 & - & - & 4 & - & 0.96 & 0.94 \\
\hline Agricultural & AG1 & $\mathrm{z}$ & 925 & - & - & 3 & - & - & $8-$ & & 3.5 & - & - & 8 & & 2.26 & 2.27 \\
\hline tract & AG2 & $\mathrm{z}$ & 722 & - & - & 2.1 & - & - & 8 & - & 2.6 & - & - & 8 & - & 1.94 & 1.91 \\
\hline Agricultural & AT1 & $\mathrm{x}$ & 335 & 51 & 27.1 & 2 & 5.2 & \begin{tabular}{|l|l}
9.7 \\
\end{tabular} & \begin{tabular}{|l|l|}
8 & 4 \\
\end{tabular} & 4 & 2.4 & 5.7 & $\overline{12}$ & \begin{tabular}{|l|l|}
4 & 4 \\
\end{tabular} & 4 & 1.62 & 1.69 \\
\hline tractor & & $\mathrm{y}$ & 728 & 165 & 27.3 & 1.1 & 3 & 7.7 & \begin{tabular}{|l|l|}
8 & 4 \\
\end{tabular} & 4 & 1.3 & 3.4 & 10 & \begin{tabular}{l|l}
8 & 4
\end{tabular} & 4 & 1.86 & 1.84 \\
\hline Articulated & AL1 & $\mathrm{x}$ & \begin{tabular}{|l|l|}
7.9 \\
\end{tabular} & 6,8 & 3.7 & 0.2 & 1.3 & 5.7 & \begin{tabular}{|l|l|}
8 & 4 \\
\end{tabular} & 4 & 1.2 & 2.3 & 7 & \begin{tabular}{|l|l|}
8 & 4 \\
\end{tabular} & 2 & 0.50 & 0.49 \\
\hline truck & & $\mathrm{y}$ & 15.7 & 21.9 & 1.8 & 0.4 & 2 & 6 & \begin{tabular}{|l|l|}
4 & 8 \\
\end{tabular} & 4 & 1.5 & 2.4 & 14 & \begin{tabular}{|l|l}
4 & 8
\end{tabular} & 2 & 0.62 & 0.63 \\
\hline
\end{tabular}


(a)

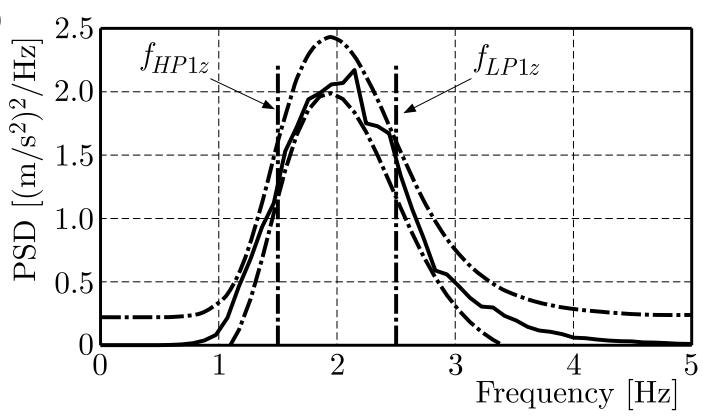

(c)

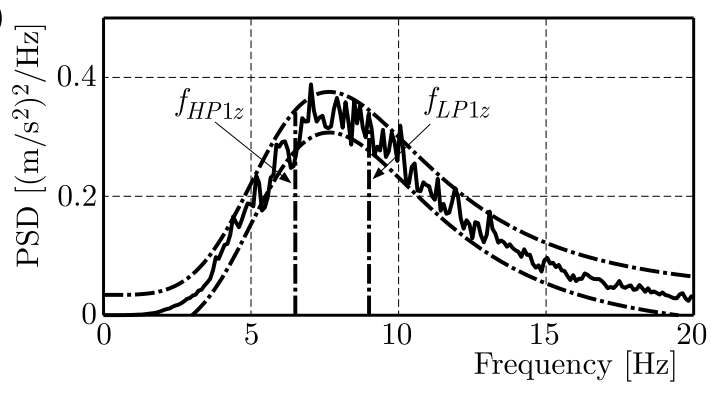

(e)

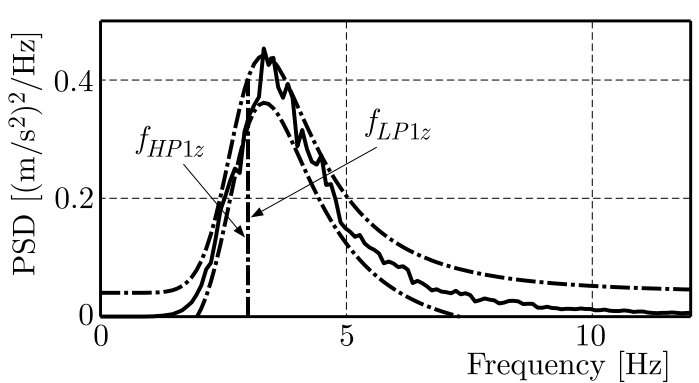

(g)

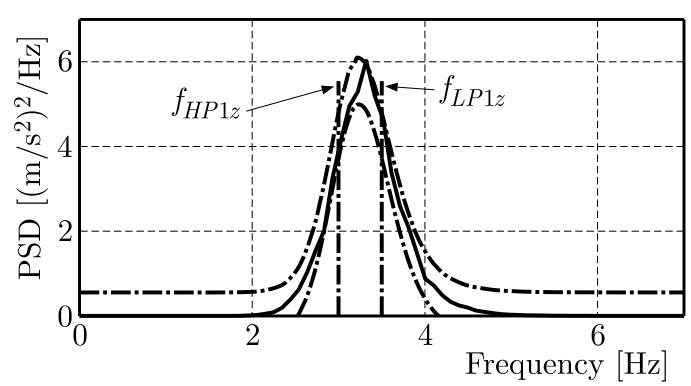

(b)

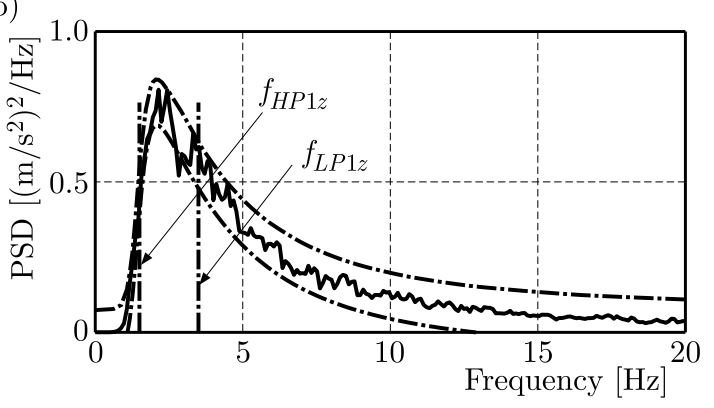

(d)

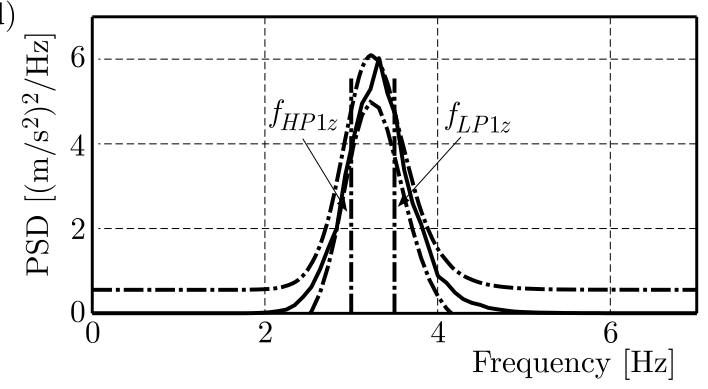

(f)

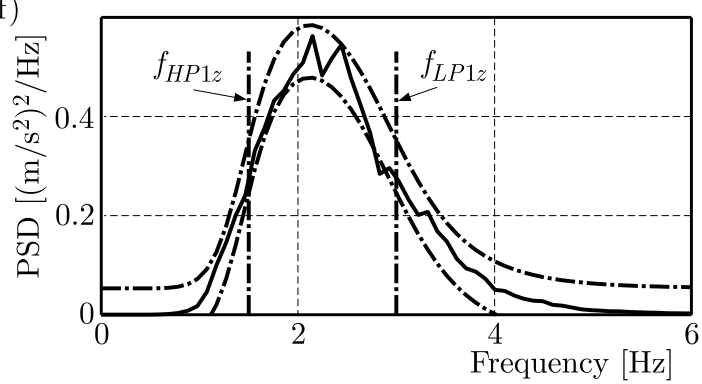

(h)

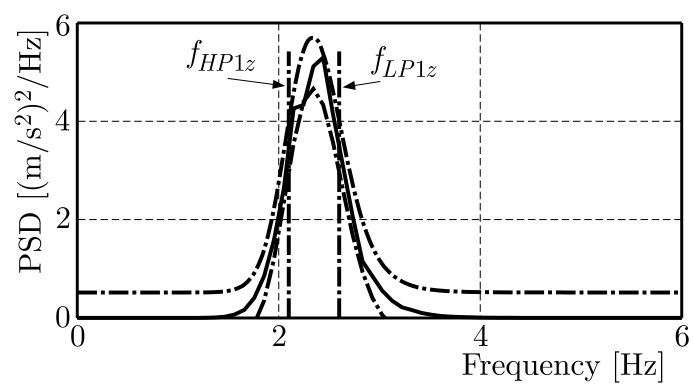

Fig. 9. Power spectral densities of simulated vertical input vibration (-) for spectral classes: EM1 (a), EM5 (b), EM6 (c), EM7 (d), IT2 (e), IT3 (f), AG1 (g), AG2 (h) and their tolerances (- - -)

\section{Horizontal vibration}

In contrary to vertical vibration, there are no standardised signals available for the horizontal directions ( $x$-axis and $y$-axis). Therefore, this paper specifies input vibrations for selected spectral classes and each class consisting of longitudinal and lateral cabin floor vibration for particular working machines performing a specific operation. In the paper by Bluthner et al. (2008), the test inputs were measured as a part of the study that has been done within the framework of the European research project VIBSEAT. They do not have sufficient magnitudes to cover the majority of actual spectra observable on the field. The determination of representative spectra represents a large amount of work which is not the purpose of this paper. 
There are no designated Butterworth filters that are desired for generating vibration along the horizontal axes. Therefore, at first, PSD functions of these signals are determined using the spectral estimation method shown in Section 5. Then the variance of random signals and the cut-off frequencies of filters are evaluated using the objective function described by Eq. (5.2). Numerical values of the design parameters are presented in Table 2. The spectral characteristics of measured, estimated and simulated input vibrations, i.e. AT1, AL1 and their tolerances for the $x$ and $y$ axes, are shown in Fig. 10. The desired and obtained root mean square values of the acceleration signal are defined in Table 2.

(a)

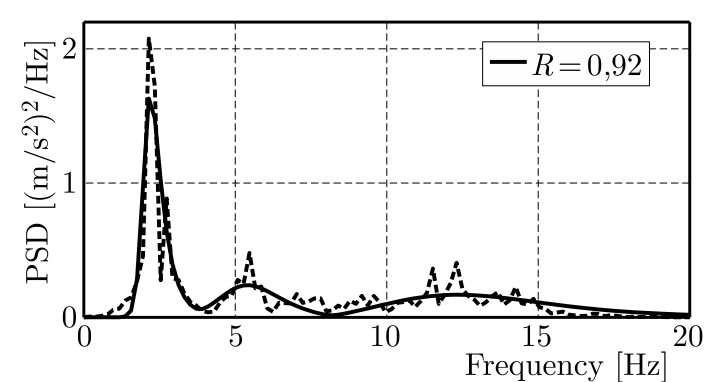

(c)

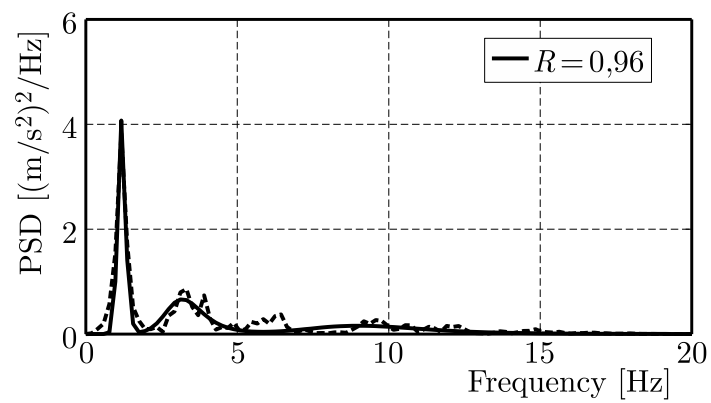

(e)

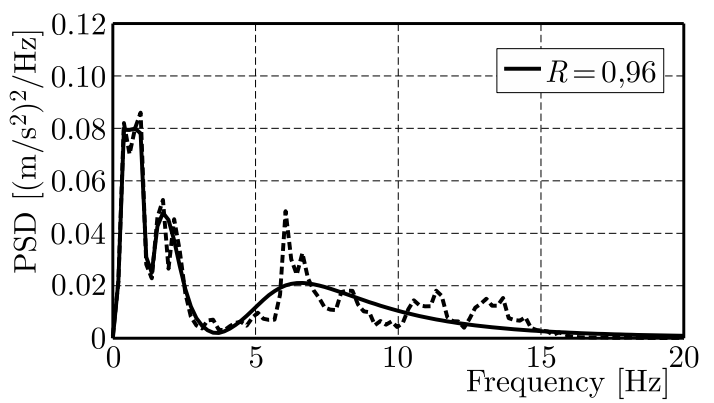

(g)

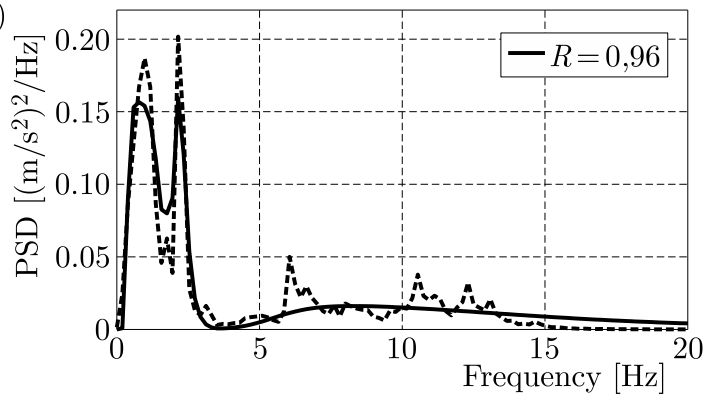

(b)

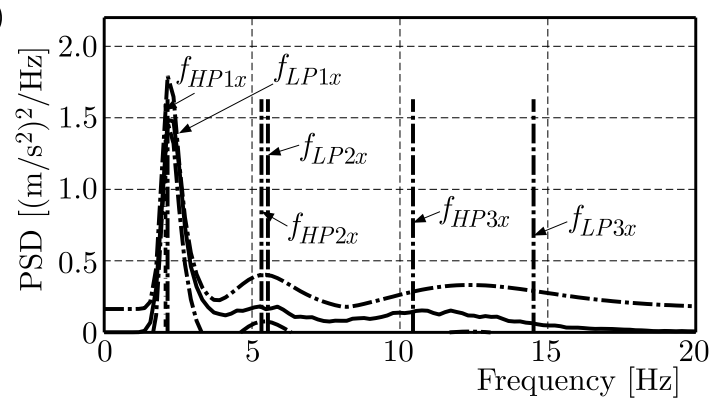

(d)
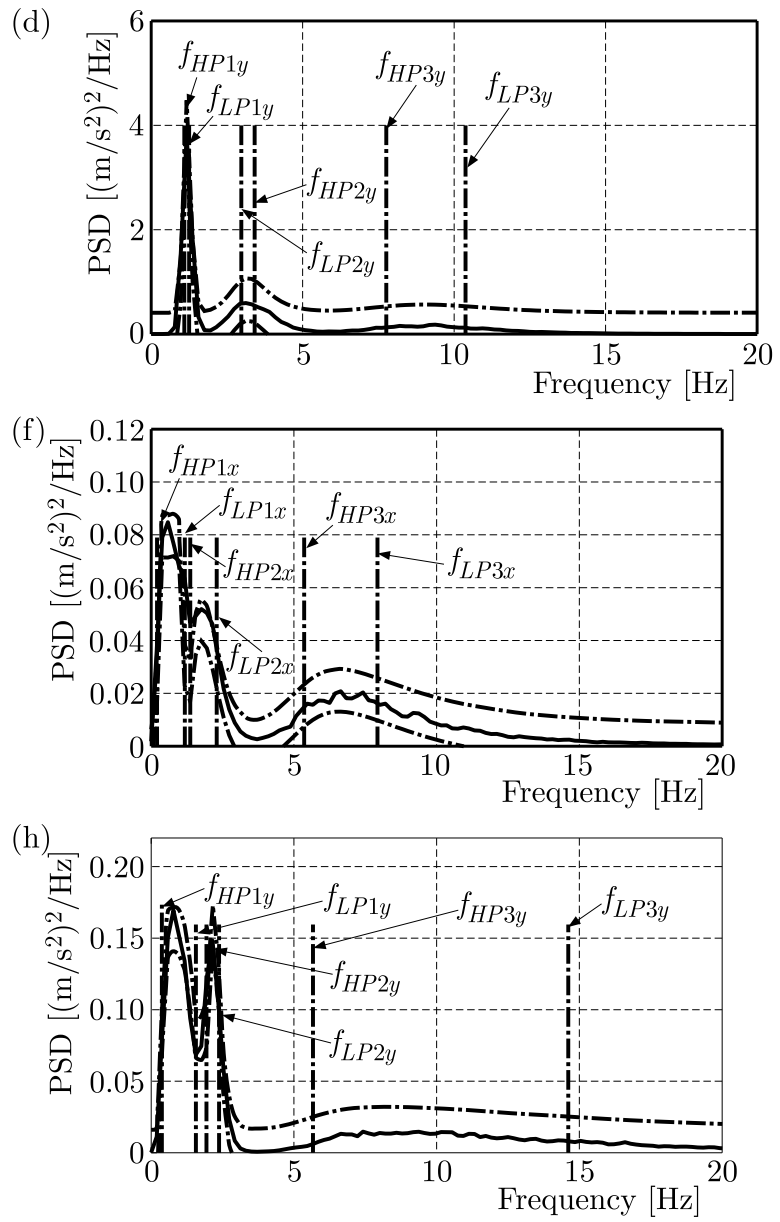

Fig. 10. Power spectral densities of measured (- - - ), estimated (-) (left-hand side) and simulated (-) (right-hand side) horizontal input vibrations for spectral classes: AT1 $x$-axis (a), (b), AT1 $y$-axis (c), (d), AL1 $x$-axis (e), (f), AL1 $y$-axis (g), (h) and their tolerances (-.-.-) 


\section{Conclusions}

In this paper, the theoretical models of simulated input vibrations are developed in such a way that the specific spectral properties of signals are obtained using the original filtration technique. This is the basis of the proposed procedure for generating random vibration that is representative for vibrations affecting the operators at work. The models developed in this paper can be used to reproduce the real working conditions of vibration reduction systems in different types of machines. Investigations carried out using the proposed signals should assist a selection process of vibro-isolation properties of systems for different spectral classes of the input vibrations.

\section{Acknowledgements}

The following work has been a part of the research project "Methods and procedures of selecting vibro-isolation properties of vibration reduction systems" funded by the National Science Center of Poland under the contract No. UMO-2013/11/B/ST8/03881.

\section{References}

1. Bendat J.S., Piersol A.G., 2004, Methods for Analysis and Measurement of Random Signals (in Polish), Polish Scientific Publishers PWN, Warsaw

2. Bluthner R., Seidel H., Hinz B., 2008, Laboratory study as basis of the development for a seat testing procedure in horizontal directions, International Journal of Industrial Ergonomics, 38, $447-456$

3. British Standards Institution BS EN 13490, 2002, Mechanical vibration - Industrial trucks - Laboratory evaluation and specification of operator seat vibration, London

4. Directive 2002/44/EC of the European Parliament and of the Council, 2002, On the minimum health and safety requirements regarding the exposure of workers to the risks arising from physical agents (vibration), Official Journal of the European Communities, 13-18

5. Griffin M.J., Howarth H.V.C., Pitts P.M., Fischer S., Kaulbars U., Donati P.M., Bereton P.F., 2006, Guide to good practice on whole-body vibration. Non-binding guide to good practice with a view to implementation of Directive 2002/44/EC on the minimum health and safety requirements regarding the exposure of workers to the risks arising from physical agents (vibrations), European Commission, Luxembourg

6. International Organization for Standardization, 1997, Mechanical vibration and shock - Evolution of human exposure to whole body vibration, ISO 2631, Genewa

7. International Organization for Standardization, 2000, Earth-moving machinery - Laboratory evaluation of operator seat vibration, ISO 7096, Genewa

8. International Organization for Standardization, 2003, Agricultural wheeled tractors - Operator's seat - Laboratory measurement of transmitted vibration, ISO 5007, Genewa

9. Kowal J., Pluta J., Konieczny J., Kot A., 2008, Energy recovering in active vibration isolation system - results of experimental research, Journal of Vibration and Control, 14, 7, 1075-1088

10. Krzyzynski T., Maciejewski I., Chamera S., 2004, Modeling and simulation of active system of truck vibroisolation with biomechanical model of human body under real excitation, VDI Publications (The Association of German Engineers), 1821, 377-390

11. Maciejewski I., Kiczkowiak T., Krzyzynski T., 2011, Application of the Pareto-optimal approach for selecting dynamic characteristics of seat suspension systems, Vehicle System Dynamics, 49, 12, 1929-1950

12. Maciejewski I., KrzyżyŃski T., 2011, Control design of semi-active seat suspension, Journal of Theoretical and Applied Mechanics, 49, 4, 1151-1168 
13. Maślanka M., SApiński B., Snamina J., 2007, Experimental study of vibration control of a cable with an attached MR damper, Journal of Theoritical and Applied Mechanics, 45, 4, 893-917

14. Nabaglo T., Kowal J., Jurkiewicz A., 2013, Construction of a parametrized tracked vehicle model and its simulation in MSC.ADAMS program, Journal of Low Frequency Noise Vibration and Active Control, 32, 1/2, 167-173

15. Parks T.W., Burrus C.S., 1987, Digital Filter Design, John Wiley \& Sons, New York

16. Preumont A., 2002, Vibration Control of Active Structures An Introduction, Kluwer Academic Publishers, London

17. Rutzel S., Hinz B., Wolfel H.B., 2006, Modal description - a better way of characterizing human vibration behavior, Journal of Sound and Vibration, 298, 810-823

18. SAPIŃski B., RosóŁ M., 2007, MR Damper performance for shock isolation, Journal of Theorethical and Applied Mechanics, 45, 1, 133-145

19. Snamina J., Kowal J., Orkisz P., 2013, Active suspension based on low dynamic stiffness, Acta Physica Polonica A, 123, 6, 1118-1122

20. Stein G.J, Muka P., Chmurny R., Hinz B., Bluthner R., 2007, Measurement and modelling of $\mathrm{x}$-direction apparent mass of the seated human body - cushioned seat system, Journal of Biomechanics, 40, 1493-1503

21. Toward M., Griffin J., 2011, The transmission of vertical vibration through seats: influence of the characteristics of the human body, Journal of Sound and Vibration, 330, 6526-6543 